\title{
A CLASS OF CONVEX BODIES( $\left.{ }^{1}\right)$
}

BY

ETHAN D. BOLKER

In this paper I show that the following four properties of a convex subset $Z$ of $\boldsymbol{R}^{n}$ are equivalent:

1. $Z$ is the range of a vector measure.

2. $Z$ is a limit of sums of segments.

3. The polar of $Z$ is a central section of the unit ball of $L^{1}$.

4. The polar of $Z$ is a projection body.

A $Z$ which enjoys one, and hence all, of the above properties is called a zonoid.

Most of the results of this paper concern the class of zonoids and do not give simple geometric conditions characterizing membership in that class. Such conditions are known only for polytopes.

Statements 1 through 4 above clearly imply that the study of zonoids belongs to several disciplines, among which are functional analysis, convexity and the geometry of polytopes. I have tried to make this paper self-contained enough to be accessible to any interested reader and have therefore restated some elementary definitions which would be unnecessary in a work directed at a specialist in one field.

I have also tried to unify the study of zonoids, so many results known to some readers appear here with new-or sometimes old-proofs. I have been as accurate as I could in assigning priorities and giving references.

1. The range of a measure. Let $\mathscr{S}$ be a $\sigma$-field of subsets of the set $X$ and $\sigma: \mathscr{S} \rightarrow \boldsymbol{R}^{n}$ be countably additive. Such a $\sigma$ will be called a vector measure; $\sigma$ is atom free if $\sigma(E) \neq 0$ implies the existence of an $F \subseteq E$ with $0 \neq \sigma(F) \neq \sigma(E)$.

1.1. Definition. A zonoid is the range of an atom free vector measure. Write $\mathscr{Z}$ for the set of zonoids.

1.2. THEOREM. The zonoid $Z=\sigma(\mathscr{S})$

1. Is compact and convex.

2. Contains 0.

3. Is symmetric about $\frac{1}{2} \sigma(X)$.

Proof. The first assertion is a well-known theorem of Liapounoff's: see [28] and [6], [22] and [30]. The second is trivial: $\sigma(\varnothing)=0 \in \sigma(\mathscr{S})$. The third follows from

$$
\sigma(E)-\frac{1}{2} \sigma(X)=-\left(\sigma(X-E)-\frac{1}{2} \sigma(X)\right) .
$$

Received by the editors September 11, 1968 and, in revised form, May 2, 1969.

( $\left.{ }^{1}\right)$ This research was supported in part by a Bryn Mawr College Junior Faculty Research Grant.

Copyright (C) 1969, American Mathematical Society 
Let $\mathscr{K}$ be the set of compact convex subsets of $\boldsymbol{R}^{n}$ which contain 0 and have a center of symmetry. If $K \in \mathscr{K}$ let $K^{\dagger}$ be the translate of $K$ which is symmetric about 0 and $\mathscr{K}^{\dagger}=\left\{K^{\dagger} \mid K \in \mathscr{K}\right\} . \mathscr{K}$ is a semigroup with respect to the usual addition of sets:

$$
K+K^{\prime}=\left\{x+x^{\prime} \mid x \in K, x^{\prime} \in K^{\prime}\right\}
$$

and $\mathscr{K}^{\dagger}$ is a subsemigroup of $\mathscr{K}$. Theorem 1.2 says $\mathscr{Z} \subseteq \mathscr{K}$. Write $\mathscr{Z}^{\dagger}$ for $\mathscr{Z} \cap \mathscr{K}^{\dagger}$; $\mathscr{Z}^{\dagger}$ consists of the zonoids symmetric about 0 . It is often more convenient to study $\mathscr{Z}^{\dagger}$ rather than $\mathscr{Z}$; the next lemma and part 3 of Theorem 1.2 imply that $K \in \mathscr{K}$ is a zonoid if and only if $K^{\dagger} \in \mathscr{Z}^{\dagger}$.

1.3. Lemma. If $z \in Z \in \mathscr{Z}$ then $Z-z \in \mathscr{Z}$. That is, any translate of a zonoid which contains 0 is a zonoid.

Proof. This improbable result follows from a trick of Halmos's [22]. If $A \in \mathscr{S}$ set

$$
\sigma^{\prime}(E)=\sigma(E-A)-\sigma(E \cap A) .
$$

Then $\sigma^{\prime}$ is an atom free vector measure and

$$
\sigma^{\prime}(\mathscr{S})=\sigma(\mathscr{S})-\sigma(A) .
$$

1.4. Lemma. The linear image of a zonoid is a zonoid. In particular, a projection of a zonoid is one.

Proof. If $T: \boldsymbol{R}^{n} \rightarrow \boldsymbol{R}^{m}$ is linear then $T \circ \sigma$ is an atom free vector measure and $T \circ \sigma(\mathscr{S})=T(\sigma(\mathscr{S}))$.

1.5. THEOREM. A sum of zonoids is a zonoid.

Proof. Suppose $(X, \mathscr{S}, \sigma)$ and $\left(X^{\prime}, \mathscr{S}^{\prime}, \sigma^{\prime}\right)$ are atom free vector measures. Let $Y$ be the disjoint union of $X$ and $X^{\prime}$ and $\tau$ the measure $\sigma+\sigma^{\prime}$ on $Y$ defined in the obvious way:

$$
\tau(E)=\sigma(E \cap X)+\sigma^{\prime}\left(E \cap X^{\prime}\right) .
$$

Then the range of $\tau$ is $\sigma(\mathscr{S})+\sigma^{\prime}\left(\mathscr{S}^{\prime}\right)$.

This argument works for countable sums too when suitably modified. If $\left\{Z_{n}\right\}_{n=1}^{\infty} \subset \mathscr{Z}$ and $\sum_{i=1}^{\infty} z_{i}$ converges for every set of choices of $z_{i} \in Z_{i}$ then $\sum_{i=1}^{\infty} Z_{i}$, defined in the obvious way, is a zonoid. We can use this fact to show that the restriction that $\sigma$ be atom free in Definition 1.1 is only a minor annoyance.

1.6. THEOREM. Let $\tau$ be a vector valued measure. Then the convex hull of the range of $\tau$ is a zonoid.

Proof. Let $x_{1}, x_{2}, \ldots$ be the atoms of $\tau$; suppose $\tau\left(\left\{x_{i}\right\}\right)=v_{i} \in \boldsymbol{R}^{n}$. Let $\tau_{0}$ be the continuous part of $\tau, \lambda$ Lebesgue measure on $[0,1]$ and, for $i \geqq 1, \tau_{i}$ the vector measure $\lambda v_{i}$ on $[0,1]$. Let $Z_{i}$ be the range of $\tau_{i}$ for $i \geqq 0$. Then $Z=\sum_{i \geqq 0} Z_{i}$ is a zonoid and is the convex hull of the range of $\tau$ since that range is closed ([22]). 
2. Moments and the support function of a zonoid. In this section we construct a large class of zonoids and reprove a result of Rickert [37] and Cunningham (unpublished) which shows that this class exhausts $\mathscr{Z}$.

Write $\langle$,$\rangle for the usual inner product on \boldsymbol{R}^{n}$ and $\|\cdot\|$ for the Euclidean norm it induces. Let $S^{n-1}$ be the unit sphere $\{x \mid\|x\|=1\}$. Since we have fixed an inner product we can identify $\boldsymbol{R}^{n}$ with its dual $\boldsymbol{R}^{n^{*}}$; in spite of this natural identification we shall try to use Latin letters for vectors and Greek letters for linear functionals. For each $\xi \in \boldsymbol{R}^{n}$ let

$$
H_{\xi}=\left\{x \in \boldsymbol{R}^{n} \mid\langle x, \xi\rangle \geqq 0\right\}
$$

be the half space on which $\xi$ is nonnegative. Then $H_{-\xi}=-H_{\xi}$ and $H_{\xi} \cap H_{-\xi}=\xi^{\perp}$.

The support function $\psi_{K}$ of $K \in \mathscr{K}$ is defined for $\xi \in R^{n}$ by

$$
\psi(\xi)=\sup \{\langle x, \xi\rangle: x \in K\}
$$

Since $K$ is closed the supremum in (2) is really a maximum. Since $0 \in K, \psi \geqq 0$. If $\|\xi\|=1 K$ is contained in the half space $H_{-\xi}+\psi(\xi)$. Since $K$ is convex it is an intersection of half spaces and hence is determined by $\psi$. When $K$ is an arbitrary bounded subset of $\boldsymbol{R}^{n}$, (2) defines the support function of the closed convex hull of $K . \psi$ is a continuous, convex function on $\boldsymbol{R}^{n}$. It is clear from (1) and (2) that adding convex sets corresponds to adding their support functions:

$$
\psi_{K+K^{\prime}}=\psi_{K}+\psi_{K^{\prime}}
$$

For these and other facts about support functions see [15, Chapter 3], or [7, Chapter 4].

Let $\mu$ be a positive, finite Borel measure on $\boldsymbol{R}^{n}$ for which every linear functional is summable. That is, for every $\xi \in \boldsymbol{R}^{n}$,

$$
\int|\langle x, \xi\rangle| d \mu(x)<\infty .
$$

Condition (4) will always be satisfied when $\mu$ has bounded support. We wish to define a vector measure $\tilde{\mu}$ by computing the moment of $\mu$ for Borel subsets $E$ of $\boldsymbol{R}^{n}$ :

$$
\tilde{\mu}(E)=\int_{E} x d \mu(x)
$$

Almost any definition of the vector valued integral in (5) will do. We shall assume $\tilde{\mu}$ is defined by the relations

$$
\langle\tilde{\mu}(E), \xi\rangle=\int_{E}\langle x, \xi\rangle d \mu(x)
$$

for every $E \subseteq \boldsymbol{R}^{n}$ and $\xi \in \boldsymbol{R}^{n}$. This definition makes sense because for fixed $E$ the right member of (6) is linear in $\xi$ and hence determines a vector $\tilde{\mu}(E) \in \boldsymbol{R}^{n}$. It is easy to see that $\tilde{\mu}$ is countably additive. Let $Z_{\mu}$ be the zonoid which is the convex hull of the range of $\tilde{\mu}$; call $Z_{\mu}$ a zonoid of moments. 
2.1. THEOREM. The support function $\psi_{\mu}$ of $Z_{\mu}$ is given by

$$
\psi_{\mu}(\xi)=\int_{H_{\xi}}\langle x, \xi\rangle d \mu(x) .
$$

Proof. Suppose $E \subseteq R^{n}$. Since $\langle x, \xi\rangle$ is nonnegative for $x \in H_{\xi}$ and nonpositive for $x \in-H_{\xi}$,

$$
\begin{aligned}
\langle\tilde{\mu}(E), \xi\rangle & =\int_{E}\langle x, \xi\rangle d \mu(x) \\
& \leqq \int_{E \cap H_{\xi}}\langle x, \xi\rangle d \mu(x) \\
& \leqq \int_{H_{\xi}}\langle x, \xi\rangle d \mu(x) \\
& =\left\langle\tilde{\mu}\left(H_{\xi}\right), \xi\right\rangle .
\end{aligned}
$$

Thus the supremum (2) which defines $\psi_{\mu}(\xi)$ has as its value the last member of (8).

2.2. Corollary. $Z_{\mu+v}=Z_{\mu}+Z_{v}$.

Proof. Combine equations (3) and (7).

2.3. LEMMA. Let $K \in \mathscr{K}$ have center of symmetry $x_{0}$. Then the support function $\psi^{\dagger}$ of $K^{\dagger}=K-x_{0}$ satisfies

$$
\psi^{\dagger}(\xi)=\psi(\xi)-\left\langle x_{0}, \xi\right\rangle=\frac{1}{2}(\psi(\xi)+\psi(-\xi)) .
$$

Proof. The first equality is a trivial consequence of (2); it tells how $\psi_{K}$ behaves when we translate $K$. To prove the second observe that $\psi^{\dagger}(\xi)=\psi^{\dagger}(-\xi)$ because $K^{\dagger}$ is symmetric about 0 . Then

$$
\begin{aligned}
2 \psi^{\dagger}(\xi) & =\psi^{\dagger}(\xi)+\psi^{\dagger}(-\xi) \\
& =\psi(\xi)-\left\langle x_{0}, \xi\right\rangle+\psi(-\xi)-\left\langle x_{0},-\xi\right\rangle \\
& =\psi(\xi)+\psi(-\xi)
\end{aligned}
$$

2.4. Corollary. The support function $\psi=\psi_{\mu}^{\dagger}$ of $Z_{\mu}^{\dagger}$ satisfies

$$
\psi=\psi_{\mu}^{\dagger}(\xi)=\frac{1}{2} \int|\langle x, \xi\rangle| d \mu(x)
$$

Proof. Note that $\langle x, \xi\rangle=0$ when $x \in H_{\xi} \cap H_{-\xi}$ and that $H_{\xi} \cup H_{-\xi}=\boldsymbol{R}^{n}$. Then

$$
\begin{aligned}
2 \psi_{\mu}^{\dagger}(\xi) & =\psi_{\mu}(\xi)+\psi_{\mu}(-\xi) \\
& =\int_{H_{\xi}}\langle x, \xi\rangle d \mu(x)+\int_{H_{-\xi}}\langle x,-\xi\rangle d \mu(x) \\
& =\int_{H_{\xi}}|\langle x, \xi\rangle| d \mu(x)+\int_{H_{-\xi}}|\langle x, \xi\rangle| d \mu(x) \\
& =\int_{H_{\xi} \cup H_{-\xi}}|\langle x, \xi\rangle| d \mu(x) .
\end{aligned}
$$


2.5. THEOREM. Every zonoid is a zonoid of moments. In fact, if $Z \in \mathscr{Z}$ then $Z=Z_{\mu}$ for a measure $\mu$ with support in $S^{n-1}$.

Proof. This is Theorem 1 of [37]. Since the proof is short we repeat it.

Suppose $Z=\sigma(\mathscr{S})$. Let $|\sigma|$ be the total variation measure of $\sigma$ and $f=d \sigma / d|\sigma|$. Then $f: X \rightarrow S^{n-1}$, at least almost everywhere modulo $|\sigma|$. Define $\mu$ on $S^{n-1}$ by $\mu=|\sigma| \circ f^{-1} ;$ then $\tilde{\mu}=\sigma \circ f^{-1}$. Thus $Z_{\mu} \subseteq Z$. However, for all $\xi \in \boldsymbol{R}^{n}$ and $A \in \mathscr{S}$

so $\psi_{z} \leqq \psi_{\mu}$ and $Z \subseteq Z_{\mu}$.

$$
\langle\sigma(A), \xi\rangle \leqq\left\langle\sigma\left(f^{-1}\left(H_{\xi}\right)\right), \xi\right\rangle
$$

As a further corollary to Theorem 2.5 note that when we combine it with Corollary 2.2 we get a new proof of Theorem 1.5.

2.6. Corollary. $K \in \mathscr{K}$ is a zonoid if and only if the support function of $K^{\dagger}$ is given by (9) for some positive measure $\mu$ on $S^{n-1}$.

The functions representable in the form given by equation (9) can be described in still another way. We call a $\psi: \boldsymbol{R}^{n} \rightarrow \boldsymbol{R}$ negative definite if for each $t>0$, $\exp (-t \psi)$ is positive definite in the classical sense and homogeneous if and only if $\psi(a \xi)=$ $|a| \psi(\xi)$ for all $a$ and $\xi$. The following theorem is due to Levy.

2.7. THEOREM. A function $\psi$ satisfies equation (9) for some positive measure $\mu$ on $S^{n-1}$ if and only if it is negative definite and homogeneous.

This elegant theorem is quoted by Choquet [11], Gelbart [19] and Herz [24]. It connects the theory of zonoids with that of Levy's symmetric stable laws.

Choquet, Rickert, Schneider and others have independently discovered how well $Z_{\mu}$ determines $\mu$. Let $P^{n-1}$ be $n-1$ dimensional projective space and $\pi: S^{n-1} \rightarrow P^{n-1}$ the natural projection defined by identifying antipodes. Let $C_{E}\left(S^{n-1}\right)$ be the subalgebra of $C\left(S^{n-1}\right)$ which contains the continuous real valued functions on $S^{n-1}$ which agree at antipodes; these are the even functions. Then the map $f \leadsto f \circ \pi$ maps $C\left(P^{n-1}\right)$ isomorphically onto $C_{E}\left(S^{n-1}\right)$. Moreover, for every measure $\mu$ on $S^{n-1}$

$$
\int_{S^{n-1}} f \circ \pi d \mu=\int_{P^{n-1}} f d\left(\mu \circ \pi^{-1}\right) .
$$

For each $\xi \in S^{n-1}$ define $g_{\xi} \in C_{E}\left(S^{n-1}\right)$ by

$$
g_{\xi}(x)=|\langle x, \xi\rangle| \text {. }
$$

2.8. THEOREM. The linear span of $\left\{g_{\xi} \mid \xi \in S^{n-1}\right\}$ is uniformly dense in $C_{E}\left(S^{n-1}\right)$.

Proof. Let $\lambda$ be the unique orthogonally invariant probability measure on $S^{n-1}$. In [40, p. 77] Schneider explicitly solves the integral equation

$$
F(\xi)=\int g_{\xi}(x) f(x) d \lambda(x)
$$

given $F \in C_{E}\left(S^{n-1}\right)$ of class $C^{n+3}$. The resulting $f$ is in $C_{E}\left(S^{n-1}\right)$. 
The Riemann sums which converge to the integral in (11) are uniform approximations to $F$ by finite linear combinations of the functions $g_{\xi}$ (see [40], p. 80). Since $C^{n+3} \cap C_{E}$ is uniformly dense in $C_{E}$ the result follows.

Schneider's solution of (11) uses the expansion of $F$ as a sum of spherical harmonics; it is a rigorous generalization of the similar but more casual assertion in Blaschke, [4, p. 154].

Theorem 2.8 is essentially equivalent to Lemma 1 of [37] and to Lemma 2 of [38], both of which Rickert proves using spherical harmonics. It can also be deduced from knowledge of the structure of the lattice of piecewise linear continuous functions on $\boldsymbol{R}^{n}$.

Equation (11) allows us to reformulate the "zonoid problem" for smooth convex bodies. If the support function $F$ of $K \in \mathscr{K}^{\dagger}$ is of class $C^{n+3}$ then $K \in \mathscr{Z}^{\dagger}$ if and only if the function $f$ in (11) is nonnegative; then Corollary 2.6 shows $K=Z_{\mu}$ when $d \mu=f d \lambda$.

2.9. Corollary ([37, Theorem 2], [11, Theorem 22]). $Z_{\mu}$ is a translate of $Z_{v}$ if and only if $\mu \circ \pi^{-1}=\nu \circ \pi^{-1}$.

Proof. $Z_{\mu}$ is a translate of $Z_{v}$ if and only if $Z_{\mu}^{\dagger}=Z_{v}^{\dagger}$, or equivalently, $\psi_{\mu}^{\dagger}=\psi_{v}^{\dagger}$. That equality is equivalent to

$$
\int_{S^{n-1}} g_{\xi}(x) d \mu(x)=\int_{S^{n-1}} g_{\xi}(x) d \nu(x)
$$

for every $\xi \in S^{n-1}$. Finally, equation (10) and Theorem 2.8 imply that (12) holds just when $\mu \circ \pi^{-1}$ and $\nu \circ \pi^{-1}$ are equal when regarded as elements of $C\left(P^{n-1}\right)^{*}$, that is, when they are the same measure. Note that this argument works in reverse too: Corollary 2.9 implies Theorem 2.8 .

The set $K \in \mathscr{K}$ is decomposable when there are sets $K_{1}$ and $K_{2} \in \mathscr{K}$ for which $K=K_{1}+K_{2}$ and such that neither $K_{1}$ nor $K_{2}$ is a translate of a homothetic copy of $K$.

\subsection{TheOREM. A zonoid which is not a line segment is decomposable.}

Proof. It follows from Corollary 2.9 and Lemma 2.4 that $Z_{\mu}$ is a line segment if and only if the measure $\mu \circ \pi^{-1}$ is concentrated at one point of $P^{n-1}$. Thus if $Z_{\mu}$ is not a line segment we can find a subset $X_{1} \subseteq P^{n-1}$ such that, setting $Y_{1}=$ $\pi^{-1}\left(X_{1}\right)$ and $Y_{2}=S^{n-1}-Y_{1}, \mu\left(Y_{1}\right) \neq 0 \neq \mu\left(Y_{2}\right)$. Let $\mu_{i}$ be $\mu$ restricted to $Y_{i}, i=1,2$. Then $\mu=\mu_{1}+\mu_{2}$ so $Z_{\mu}=Z_{\mu_{1}}+Z_{\mu_{2}}$ (Corollary 2.2), but it is clear from Corollary 2.9 that neither $Z_{\mu_{1}}$ nor $Z_{\mu_{2}}$ is the translate of a homothetic copy of $Z_{\mu}$.

Theorem 2.10 implies that a polytope all of whose two dimensional faces are triangles is not a zonoid, for such polytopes are indecomposable (Shephard, [44, p. 92]). In the next section we shall show such polytopes, and others, are not zonoids without showing they are indecomposable.

We really proved more than we stated in Theorem 2.10 . Partition $P^{n-1}$ into 
sets $X_{1}, \ldots, X_{N}$ of "small diameter". The corresponding partition $\left\{\pi^{-1}\left(X_{i}\right)\right\}$ of $S^{n-1}$ and $\mu=\mu_{1}+\cdots+\mu_{N}$ leads to the decomposition

$$
Z_{\mu}=Z_{\mu_{1}}+\cdots+Z_{\mu_{N}}
$$

Here each $Z_{\mu_{i}}$ is "spindly": $\pi^{-1}\left(X_{i}\right)$ is a disjoint union $Y_{i} \cup\left(-Y_{i}\right)$ and $Z_{\mu_{i}}$ has vertices at $\pm \mu_{i}\left(Y_{i}\right)$ whose sharpness increases as the diameter of $Y_{i}$ decreases. When $Y_{i}$ is an atom of $\mu, Z_{\mu_{i}}$ is a segment. Thus every zonoid is a sum of almostsegments. These remarks will reappear in formal disguise in $\$ 5$, where we shall prove that every zonoid is almost a sum of segments.

3. Zonotopes. The faces of a $K \in \mathscr{K}$ are the intersections of $K$ with its support hyperplanes. Each face is a compact convex set of dimension $\leqq n-1$. If $K$ is of dimension $<n$ it will be a face of itself.

When $\xi \in S^{n-1}$

$$
F_{\xi}=F_{\xi}(K)=\{x \in K \mid\langle x, \xi\rangle=\psi(\xi)\}
$$

is the face of $K$ in (one of) the (at most two) support hyperplane(s) parallel to $\xi^{\perp}$. It follows from (3) and (13) that

$$
F_{\xi}\left(K+K^{\prime}\right)=F_{\xi}(K)+F_{\xi}\left(K^{\prime}\right)
$$

[7, p. 31].

Let $V$ be a proper subspace of $\boldsymbol{R}^{n}$ and $\mu_{V}$ the restriction of $\mu$ to the Borel subsets of $V$. We may also consider $\mu_{V}$ a measure on all of $\boldsymbol{R}^{n}$. Then $\mu-\mu_{V}$ is nonnegative on $\boldsymbol{R}^{n}$ and 0 on $V$. Since $Z_{\mu_{V}}$ is a zonoid in $V$ it has dimension $\leqq n-1$. Since

$$
Z_{\mu}=Z_{\mu-\mu_{V}}+Z_{\mu_{V}}
$$

(Corollary 2.2) it follows from (13) that $Z_{\mu_{V}}$ is a summand of $F_{\xi}$ whenever $V \subseteq \xi^{\perp}$. We are about to strengthen these informal observations by showing that proper faces of $Z_{\mu}$ correspond to proper subspaces of $\boldsymbol{R}^{n}$ of positive $\mu$-measure.

3.1. Lemma. $F_{\xi}\left(Z_{\mu}\right)$ is the convex hull of the points $\tilde{\mu}(E)$ for $H_{\xi}^{\circ} \subseteq E \subseteq H_{\xi}\left(H_{\xi}^{\circ}\right.$ is the interior of $H_{\xi}$; it is an open half space).

Proof. Examine the inequalities in (8) to see when they are strict. They show that for $H_{\xi}^{\circ} \subseteq E \subseteq H_{\xi}$,

$$
\langle\mu(E), \xi\rangle=\psi_{\mu}(\xi)
$$

Conversely, (16) implies $H_{\xi}^{\circ} \subseteq E \subseteq H_{\xi}$ to within $\mu$-null sets.

3.2. THEOREM. Every face of the zonoid $Z_{\mu}$ is a translate of a zonoid of lower dimension which is a summand of $Z_{\mu}$.

Proof. Let $V=\xi^{\perp}$. Then Lemma 3.1 implies

$$
F_{\xi}=\mu\left(H_{\xi}^{\circ}\right)+Z_{\mu_{V}} .
$$

We observed in (15) that $Z_{\mu_{V}}$ is a summand of $Z_{\mu}$. 
3.3. COROLlaRY. $Z_{\mu}$ has a proper face in a coset of $V$ if and only if $\mu_{V} \neq 0$.

Proof. Since the relation "is a face of" is transitive we may apply the argument in Theorem 3.2 inductively.

We can now identify many nonzonoids without resorting to indecomposability arguments. Theorem 3.2 implies that one face which is not centrally symmetric suffices to disqualify a prospective zonoid. Thus the octahedron, or, more generally, the $n$ dimensional cross polytope

$$
l_{n}^{1}=\left\{\left(x_{1}, \ldots, x_{n}\right):\left|x_{1}\right|+\cdots+\left|x_{n}\right| \leqq 1\right\}
$$

is not a zonoid. Neither is a generalized cross polytope, a continuous one-to-one linear image of a cross polytope.

The double cone $\left\{(x, y, z):\left(x^{2}+y^{2}\right)^{1 / 2}+|z| \leqq 1\right\}$ obtained by rotating a square about a diagonal is not a zonoid. Although its proper faces are line segments, which are zonoids, they do not appear as summands. This cone also happens to be indecomposable.

A trapezoid which is not a parallelogram is decomposable but is not a zonoid. It is not centrally symmetric. A truncated octahedron $K$ is centrally symmetric and decomposable but will not be a zonoid unless the truncation points divide the edges of the octahedron into three equal parts so that the hexagonal faces of $K$ have centers of symmetry.

We now have the machinery to describe the zonotopes, those zonoids which happen to be polytopes. They have a long history which begins with Kepler. The crystallographer Fedorov seems to have discovered them as a class. Parts of the next theorem are due (in part independently) to Alexandrov [1], Blaschke [5, p. 81] and [3, p. 250], Burckhardt [8], Coxeter [12], [13], Cunningham, Lindquist [32], Rickert, Shephard [46] and myself, and, presumably, many others. The critical implication $(5 \Rightarrow 3)$ below is implicit in the well-known construction of a "zone" of faces of a zonohedron [13, p. 27] though I have not seen it written down in this form anywhere. For more history consult [12] and [46].

Some parts of the theorem below are false as stated. To correct them "a translate of" must be inserted in the appropriate places. I have omitted the phrase in order to make the statements less cluttered.

3.3. THEOREM. The following conditions on a convex polytope $K \subseteq \boldsymbol{R}^{n}$ are equivalent:

1. $K$ is a zonotope.

2. $K=Z_{\mu}$ for an atomic measure $\mu$.

3. $K$ is a sum of line segments.

4. $K$ is a linear image of the unit cube in $\boldsymbol{R}^{N}$ for some $N$.

5. Every $n-1$ dimensional face of $K$ is a zonotope $(n \geqq 3)$.

6. For some $r$ with $2 \leqq r \leqq n$, every $r$ dimensional face of $K$ is a zonotope.

7. Every two dimensional face of $K$ is centrally symmetric. 
Proof. We shall show

$$
\begin{aligned}
& 1 \Rightarrow 2 \Rightarrow 3 \Rightarrow 4 \Rightarrow 1 \\
& \Downarrow \quad \Uparrow \\
& 7 \Rightarrow 6 \Rightarrow 5
\end{aligned}
$$

The hardest step is $(5 \Rightarrow 3)$, which we shall leave for last.

$(1 \Rightarrow 2)$. Suppose $K$ is a zonotope. Then $K=Z_{\mu}$ for some $\mu$. Since every edge of $K$ is a face of $K$, Theorem 3.2 shows $\mu$ is supported by a finite number of one dimensional subspaces of $\boldsymbol{R}^{n}$. Since it is supported by $S^{n-1}$ it is atomic.

$(2 \Rightarrow 3)$. Let $x_{1}, \ldots, x_{N} \in S^{n-1}$ be the atoms of $\mu$; suppose $\mu\left(\left\{x_{i}\right\}\right)=\lambda_{i}$. Then

$$
Z_{\mu}=\left[0, \lambda_{1} x_{1}\right]+\cdots+\left[0, \lambda_{N} x_{N}\right] .
$$

$(3 \Rightarrow 4)$. Suppose $K$ is given by the right member of $(18)$. Let $e_{1}, \ldots, e_{N}$ be the usual basis of $\boldsymbol{R}^{N}$ and define $T: \boldsymbol{R}^{N} \rightarrow \boldsymbol{R}^{n}$ by $T\left(e_{i}\right)=\lambda_{i} x_{i}$. Then $K$ is the image of the cube $\left\{\left(y_{1}, \ldots, y_{N}\right) \mid 0 \leqq y_{i} \leqq 1\right\}$ in $\boldsymbol{R}^{N}$.

$(4 \Rightarrow 1)$. The unit cube in $\boldsymbol{R}^{N}$ is the sum of the segments $\left[0, e_{i}\right]$, each of which is a zonoid. Hence the cube is itself a zonoid (Theorem 1.5) and so is any linear image (Lemma 1.4).

$(1 \Rightarrow 7)$. Suppose $K$ is a zonotope. Then every face of $K$ is a zonotope (Theorem 3.2) and hence centrally symmetric. Thus, a fortiori, the two dimensional faces of $K$ are centrally symmetric.

$(7 \Rightarrow 6)$. It is geometrically obvious (and proved below) that central symmetry is necessary and sufficient for a polygon (two dimensional polytope) to satisfy 3 and hence 1 above. Thus every centrally symmetric polygon is a zonogon and $7 \Rightarrow 6$.

$(6 \Rightarrow 5)$. The argument in this case depends on the implication $(5 \Rightarrow 3)$ which we prove below noncircularly. Suppose $(5 \Rightarrow 3 \Rightarrow 1)$ and that $K$ satisfies 6 for some $r$. If $r=n-1$ there is nothing to prove, so suppose $r<n-1$. Then every $r+1$ dimensional face of $K$ satisfies 5 and hence 1 , so $K$ satisfies 6 for $r+1$. The argument clearly continues inductively to show that $K$ satisfies 5 , which is just 6 for $r=n-1$.

To prove $(5 \Rightarrow 3)$ we need a lemma which allows us to decide when a bounded convex set $K$ has a segment as a summand. The argument below formalizes Coxeter's "contraction" of zonohedra in $[12, \S 8]$. Let $v \in S^{n-1}$ be a direction and $P$ the orthogonal projection along $v$ onto $v^{\perp}$. Describe $K$ by giving its "top" and "bottom" hypersurfaces over $P K$. That is, for each $y \in P K$ let $L(y)=K \cap P^{-1}(y)$, the chord of $K$ parallel to $v$ which when extended passes through $y$. Let

$$
\begin{aligned}
& F(y)=\sup \{\langle x, v\rangle: x \in L(y)\}, \\
& G(y)=\inf \{\langle x, v\rangle: x \in L(y)\} .
\end{aligned}
$$

Then $F$ and $-G$ are convex functions on the convex set $P K$ and

$$
K=\{x \mid P x \in P K \text { and } G(P x) \leqq\langle x, v\rangle \leqq F(P x)\} .
$$

$($ See $[4, \S 16]$.) 
Let $\lambda_{0}=\lambda_{0}(K)=\min \{F(y)-G(y) \mid y \in P K\}$. The convexity of $K$ implies that $\lambda_{0}$ is assumed for some $y$ on the boundary $\partial P K$ in $v^{\perp}$.

3.4. LEMMA. $K$ has a segment summand of length $\lambda$ parallel to $v$ if and only if $\lambda \leqq \lambda_{0}$. If

$$
K=K^{\prime}+\left[0, \lambda_{0} v\right]
$$

then $\lambda_{0}\left(K^{\prime}\right)=0$ so $K^{\prime}$ has no nontrivial summand parallel to $v$.

Proof. Suppose

$$
K=K^{\prime}+[0, \lambda v]
$$

Then $P v=0$ so $P K=P K^{\prime}$. Given $y \in P K$ choose an $x \in K^{\prime} \cap P^{-1}(y)$. Then $x^{\prime}$ and $x^{\prime}+\lambda v$ are in $K$ so

$$
F(y) \geqq\left\langle x^{\prime}+\lambda v, v\right\rangle=\left\langle x^{\prime}, v\right\rangle+\lambda \geqq G(y) .
$$

Hence $F(y)-G(y) \geqq \lambda$ for all $y$, so $\lambda_{0} \geqq \lambda$. To prove the converse it clearly suffices to find a $K^{\prime}$ for which (20) is true. Let $F^{\prime}=F-\lambda_{0}$ and $G^{\prime}=G$. Then $F^{\prime}$ and $-G^{\prime}$ are both convex and $F^{\prime} \geqq G^{\prime}$ so we can use (19) with primed $F$ and $G$ to define a convex body $K^{\prime}$. It is easy to check that this $K^{\prime}$ works in (20). Clearly

$$
\lambda_{0}\left(K^{\prime}\right)=\min \left(F^{\prime}(y)-G^{\prime}(y)\right)=0 .
$$

Now we return to the proof of the theorem. Let $K$ be a polytope satisfying condition 5. Let $v$ be an edge of $K$; we may suppose $\|v\|=1$. We shall show that $\lambda_{0}=\lambda_{0}(K, v)=1$ and that when we use Lemma 3.4 the polytope $K^{\prime}$ in (20) still satisfies 5 . Since $K^{\prime}$ has fewer edges than $K$ it follows by induction on the number of edges that $K$ is a sum of segments.

Let $F_{1}, \ldots, F_{m}$ be the hyperfaces $(=n-1$ dimensional faces $)$ of $K$. Each $F_{i}$ determines a half space $E_{i}$ such that $K=\bigcap E_{i}$. Let $M$ be the union of those hyperfaces which have an edge of length 1 parallel to $v ; M$ is a zone of hyperfaces. We may suppose $M=\bigcup\left\{F_{1}, \ldots, F_{r}\right\}$. Each $F_{i} \in M$ has $v$ for a summand since we assume $K$ satisfies 5 and we know $(1 \Rightarrow 3)$.

Each $x \in M$ lies on a support hyperplane $G$ of $K$ parallel to $v$ so $P x$ lies on the support hyperplane $P G$ of $P K$ in $v^{\perp}$. Thus $P M \subseteq \partial P K$. We wish to show $P M$ is all of $\partial P K$. To do so it suffices to show $P M$ is open and closed in $\partial P K$ since $\partial P K$ is connected: it is the boundary of $P K$ in $v^{\perp}$ and thus is homeomorphic to $S^{n-2}$, and $n \geqq 3$. $M$ is clearly compact so $P M$ is compact and hence, a fortiori, closed in $\partial P K$.

Now suppose $y \in P M$. Choose $x \in M$ such that $x \pm v / 2 \in M$ and $P x=y$; this is possible because every $F_{i} \subseteq M$ has $v$ as a summand. Then every $F_{i}$ which contains $x$ lies in $M$ so we can choose $\varepsilon$ small enough to guarantee that the open $\varepsilon$-ball $U$ about $x$ is disjoint from the complement in $\partial K$ of the zone $M$. Then

$$
U \cap K=U \cap E_{1} \cap \cdots \cap E_{r}
$$

so

$$
y \in P U \cap \partial P K=P(U \cap \partial K)=P(U \cap M) \subseteq P M
$$


Since $P$ is an open map (21) shows $y$ has a neighborhood in $\partial P K$ contained in $P M$, which is thus both open and closed. Therefore $P M=\partial P K$.

The implication $(5 \Rightarrow 3)$ we are working on is false when $n=2$ but the argument we are giving will prove that every centrally symmetric polygon $K$ is a sum of segments and thus fill the gap we left in the proof of $(7 \Rightarrow 6)$. When $v$ is an edge of such a polygon $\partial P K$ is no longer connected (it contains just two points) but $P M=\partial P K$ because the zone $M$ is the union of $v$ and its equal opposite edge.

In either case suppose $y \in \partial P K$. Then $y \in P F_{i}$ for some $F_{i} \in M$. Since $v$ is a summand of $F_{i}$, Lemma 3.4 implies $\lambda(y) \geqq 1$. Hence $\lambda_{0}(K) \geqq 1$ and $K$ has $v$ for a summand. Write $K=K^{\prime}+[0, v]$. The hyperfaces of $K^{\prime}$ are translates of the hyperfaces $F_{i} \notin M$ and summands $F_{i}^{\prime}$ of the hyperfaces $F_{i}=F_{i}^{\prime}+[0, v] \in M$. In either case they are sums of segments so $K^{\prime}$ satisfies 5 .

4. The radius of a zonoid. In this section we estimate the size of $Z_{\mu}$ in terms of $\mu\left(S^{n-1}\right)$. The first estimate is crude and easy. The second, due to Rickert and Kaufman, is sharp. The maximum radius $\rho=\rho_{K}$ of $K \in \mathscr{K}$ is $\rho=\sup \{\|x\|: x \in K\}$. Since $K$ is closed the supremum is a maximum so we can always find a radius vector $x_{0} \in K$ for which $\left\|x_{0}\right\|=\rho$. The maximum radius depends on the position of $0 \in K$ : we always have

$$
\rho_{K}^{\dagger} \leqq \rho_{K}
$$

In general, radii are not perpendicular to tangents. The geometrical content of the following lemma is that radius vectors are.

4.1. LEMMA.

$$
\psi\left(x_{0} / \rho\right)=\rho=\sup \{\psi(\xi):\|\xi\|=1\}
$$

Proof. Since $\left\|x_{0} / \rho\right\|=1$,

$$
\begin{aligned}
\psi\left(x_{0} / \rho\right) & \leqq \sup _{\|\xi\|=1} \psi(\xi) \\
& =\sup _{\|\xi\|=1} \sup _{x \in K}\langle x, \xi\rangle \\
& \leqq \sup _{\|\xi\|=1} \sup _{x \in K}\|x\|\|\xi\| \\
& =\rho=\left\|x_{0}\right\|=\left\langle x_{0} / \rho, x_{0}\right\rangle \\
& \leqq \sup _{x \in K}\left\langle x_{0} / \rho, x\right\rangle=\psi\left(x_{0} / \rho\right) .
\end{aligned}
$$

Write $\rho_{\mu}$ for the maximum radius of $Z_{\mu}$.

4.2. THEOREM. There are constants $Q$ and $Q^{\prime}$ depending only on the dimension $n$ such that

$$
Q^{\prime} \rho_{\mu} \leqq \mu\left(S^{n-1}\right) \leqq Q \rho_{\mu}
$$

for all $\mu$. 
Proof. Suppose $\|\xi\|=1$. Then

$$
\psi_{\mu}(\xi)=\int_{H_{\xi}}\langle x, \xi\rangle d \mu(x) \leqq \mu\left(S^{n-1}\right) .
$$

Take the supremum of (24) over all $\xi \in S^{n-1}$ to deduce $\rho_{\mu} \leqq \mu\left(S^{n-1}\right)$. That is, we may take $Q^{\prime}=1$ in the first inequality in (23).

To prove the second let $C(\xi)$ be the spherical cap

$$
C(\xi)=\left\{x \in S^{n-1} \mid\langle x, \xi\rangle \geqq \frac{1}{2}\right\} .
$$

Then

$$
\begin{aligned}
\psi_{\mu}(\xi) & \geqq \int_{C(\xi)}\langle x, \xi\rangle d \mu(\xi) \\
& \geqq \frac{1}{2} \mu(C(\xi)) .
\end{aligned}
$$

Since $S^{n-1}$ is compact it is covered by finitely many caps $C\left(\xi_{1}\right), \ldots, C\left(\xi_{N}\right)$ and

$$
\begin{aligned}
\mu\left(S^{n-1}\right) & \leqq \sum \mu\left(C\left(\xi_{i}\right)\right) \leqq 2 \sum \psi_{\mu}\left(\xi_{i}\right) \\
& \leqq 2 N \max _{i} \psi_{\mu}\left(\xi_{i}\right) \leqq 2 N \rho_{\mu}
\end{aligned}
$$

so $Q=2 N$ will do.

4.3. It is easy to see that 1 is the best (i.e. largest) value for $Q^{\prime}$, because

$$
\rho_{\mu}=\mu\left(S^{n-1}\right)
$$

if and only if $\mu$ is atomic with a single atom, that is, when $Z_{\mu}$ is a line segment with 0 at one end. The best (i.e., smallest) value for $Q$ has been known for years and published often. It is discussed in various disguises by Grünbaum [20], Kaufman and Rickert [27], Mayer [33], Rutovitz [39] and Semadeni [43]. Rickert [37] and others found those $\mu$ for which the minimum value of $Q$ is attained. The argument below is an analytic version of Schwarz's [42].

We are looking for the minimum $Q$. In light of (22) it suffices to look at elements of $\mathscr{Z}^{\dagger}$ and hence at zonoids whose support function is given by (9). Let $\lambda$ be the unique orthogonally invariant probability measure on $S^{n-1} ; \lambda$ is normalized "surface area" measure. Compute the average value $A$ of $\psi_{\mu}$ on $S^{n-1}$ :

$$
\begin{aligned}
A & =\int_{S^{n-1}} \psi_{\mu}(\xi) d \lambda(\xi)=\frac{1}{2} \int_{S^{n-1}} d \lambda(\xi) \int_{S^{n-1}}|\langle x, \xi\rangle| d \mu(x) \\
& =\int_{S^{n-1}} d \mu(x) \int_{S^{n-1}} \frac{1}{2}|\langle x, \xi\rangle| d \lambda(\xi) .
\end{aligned}
$$

The inner integral in (25) is independent of $x$ because $\lambda$ is rotation invariant. Its value is just the constant radius $\rho_{\lambda}$ of the ball $Z_{\lambda}$, which is easily computed:

$$
\rho_{\lambda}=\frac{1}{2 \pi^{1 / 2}} \frac{\Gamma(n / 2)}{\Gamma((n+1) / 2)} .
$$


The computation of $\rho_{\lambda}$ is cleverly simplified by Bonnesen and Fenchel [7, p. 48 and p. 2].

Lemma 4.1 now implies $\rho_{\mu} \geqq A=\mu\left(S^{n-1}\right) \rho_{\lambda}$ so that when $Q=1 / \rho_{\lambda}$ we have $\mu\left(S^{n-1}\right) \leqq Q \rho_{\mu}$ for all $\mu$ with equality if and only if $\psi_{\mu}$ is constant or, equivalently, $Z_{\mu}=Z_{\mu}^{\dagger}$ is a ball. Corollary 2.7 shows that happens if and only if $\mu \circ \pi^{-1}=\lambda \circ \pi^{-1}$ and $\tilde{\mu}\left(S^{n-1}\right)=0$. We have reproved Theorem 1 of [38].

5. $\mathscr{Z}$ as a semigroup. We observed in $\S 1$ that $\mathscr{Z}$ is a subsemigroup of $\mathscr{K}$. In this section we introduce topological considerations. Equip $\mathscr{K}$ with the customary (Hausdorff) metric: $d\left(K_{1}, K_{2}\right)=\inf \left\{\varepsilon \mid\right.$ the $\varepsilon$ neighborhood of $K_{i}$ includes $K_{j}$, $i, j=1,2\} . d$ is an invariant metric on $\mathscr{K}$.

Blaschke's famous selection theorem [15, p. 64] asserts that every net of elements of $\mathscr{K}$ with bounded maximal radii has a convergent subnet.

Let $\mathscr{M}=\mathscr{M}^{+}\left(S^{n-1}\right)$ be the cone of positive measures on $S^{n-1}$ equipped with the weak-* topology it acquires as a subset of $C\left(S^{n-1}\right)^{*}$. The total variation norm of $\mu \in \mathscr{M}$ is easy to compute. Because $\mu$ is positive, $\|\mu\|=\mu\left(S^{n-1}\right)$. Every net of elements of $\mathscr{M}$ of bounded norm has a (weak-*) convergent subnet [14, p. 424]. Write $Z$ for the map $\mu \sim Z_{\mu}$ from $\mathscr{M}$ to $\mathscr{K}$. Corollary 2.2 says $Z$ is a semigroup homomorphism. The next elementary lemma allows us to show it is continuous.

5.1. Lemma. Let $\left\{t_{\alpha}\right\}_{\alpha \in A}$ be a net in the topological space $\mathscr{T}$. Then $t_{\alpha} \rightarrow t \in \mathscr{T}$ if and only if every subnet has a subsubnet which converges to $t$.

Proof. "Only if" is obvious and not very useful. To prove the converse let $N$ be a neighborhood of $t$. Let $B=\left\{\beta \in A \mid t_{\beta} \notin N\right\}$; we must show that there is an $\alpha_{0} \in A$ for which $\beta \ngtr \alpha_{0}$ for all $\beta \in B$. But if there were no such $\alpha_{0}, B$ would be cofinal. Then $\left\{t_{\beta}\right\}_{\beta \in B}$ would have a subnet converging to $t$, which is impossible.

5.2. TheOREM. $Z: \mathscr{M} \rightarrow \mathscr{K}$ is continuous.

Proof. Suppose the net $\mu_{\alpha}$ converges to $\mu$ in the weak-* topology on $\mathscr{M}$. We must show that $Z_{\mu_{\alpha}} \rightarrow Z_{\mu}$. By virtue of Lemma 5.1 it suffices to show that every subnet $\left\{\mu_{\beta}\right\}_{\beta \in B}$ has a subnet $\left\{\mu_{\gamma}\right\}_{\gamma \in \Gamma}$ for which $Z_{\mu_{\gamma}} \rightarrow Z_{\mu}$. We know $\mu_{\beta} \rightarrow \mu$ so

$$
\begin{aligned}
\left\|\mu_{\beta}\right\| & =\mu_{\beta}\left(S^{n-1}\right) \\
& =\int 1 d \mu_{\beta} \rightarrow \int 1 d \mu=\|\mu\| .
\end{aligned}
$$

Thus we may assume $\left\{\left\|\mu_{\beta}\right\|\right\}$ is bounded.

Theorem 4.2 implies $\left\{\rho_{\mu_{\beta}}\right\}$ is bounded, so the Blaschke selection theorem allows us to extract a cofinal $\Gamma \subseteq B$ and a $K \in \mathscr{K}$ such that $Z_{\mu_{y}} \rightarrow K$. Let $\psi$ be the support function of $K$. Then $\psi_{\mu_{y}} \rightarrow \psi$ pointwise on $\boldsymbol{R}^{n}$. For each $\xi \in \boldsymbol{R}^{n}$ the function

$$
h_{\xi}(x)=\max (0,\langle x, \xi\rangle)
$$


is continuous on $S^{n-1}$ so

$$
\begin{aligned}
\psi_{u_{\gamma}}(\xi) & =\int_{H_{\xi}}\langle x, \xi\rangle d \mu_{\gamma}(x) \quad \text { (Theorem 2.1) } \\
& =\int_{S^{n-1}} h_{\xi}(x) d \mu_{\gamma}(x) \\
& \rightarrow \int_{S^{n-1}} h_{\xi}(x) d \mu(x)=\psi_{\mu}(\xi) .
\end{aligned}
$$

That is, $\psi_{\mu_{\gamma}} \rightarrow \psi_{\mu}$ pointwise. Consequently $\psi_{\mu}=\psi$ and hence $Z_{\mu}=K$.

5.3. THEOREM. $\mathscr{Z}$ is the closure in $\mathscr{K}$ of the subsemigroup of zonotopes.

Proof. It is clear from part 3 of Theorem 3.3 that the zonotopes form a subsemigroup of $\mathscr{Z}$. Part 2 of that theorem shows that the zonotopes are the images under $Z$ of the atomic measures in $\mathscr{M}$. Those measures are weak-* dense in $\mathscr{M}$ and $Z$ is continuous so $\mathscr{Z}$ is contained in the closure of the semigroup of zonotopes. The theorem will have been proved once we give the routine compactness argument which shows that $\mathscr{Z}$ is closed. Suppose $\left\{Z_{\mu_{n}}\right\}$ is Cauchy in $\mathscr{K}$. (Sequences suffice since $\mathscr{K}$ is a metric space.) Then $\left\{\rho_{\mu_{n}}\right\}$ and hence $\left\{\left\|\mu_{n}\right\|\right\}$ is bounded (Theorem 4.2) so there is a subsequence $\left\{\mu_{n_{j}}\right\}$ and a $\mu \in \mathscr{M}$ such that $\mu_{n_{j}} \rightarrow \mu$. Theorem 5.2 implies $Z_{\mu_{n}} \rightarrow Z_{\mu}$, so our original Cauchy sequence also has $Z_{\mu} \in \mathscr{Z}$ as its limit.

Theorem 5.3 shows that the zonoid problem is equivalent to a classical approximation problem in convex body theory: find the limits of the sums of segments. In this form the problem seems to have been first posed by Blaschke ([4, p. 154], [3, p. 250] and [5, pp. 81-82]), and restated by Bonnesen and Fenchel [7, p. 29]. Shephard took up a more general problem in [44] where he proved results which include the next theorem, which also appears in Schneider, [40]. Their methods differ from each others' and ours.

5.4. THEOREM. $\mathscr{Z}=\mathscr{K}$ when $n=2$. $\mathscr{Z}$ is nowhere dense in $\mathscr{K}$ when $n \geqq 3$.

Proof. Suppose $n=2$. We know every centrally symmetric polygon is a zonogon (part 3 or part 7 of Theorem 3.3). But every centrally symmetric plane convex set is clearly a limit of such polygons, so $\mathscr{Z}=\mathscr{K}$.

Suppose $n \geqq 3, \varepsilon>0$ and $Z_{\mu} \in \mathscr{Z}$. We can alter $Z_{\mu}$ by less than $\varepsilon$ to create a $K \in \mathscr{K}$ with a face which is not centrally symmetric. Theorem 3.2 implies $K \notin \mathscr{Z}$. Thus $\mathscr{Z}$ is nowhere dense. The same fact follows from another related argument of Shephard's: polytopes with triangular faces are dense in $\mathscr{K}$ and are indecomposable and hence not zonoids (Theorem 2.8).

Herz proved the first part of Theorem 5.4 in [24, p. 672], using Corollary 2.6 and some elementary differential geometry. He then showed that the octahedron is not a zonoid, using similar methods.

6. Subspaces of $L^{1}$. Throughout this section we restrict our attention to elements of $\mathscr{K}^{\dagger}$ which have nonempty interiors. Given such a $K$ let $B(K)$ be the $n$ dimensional 
Banach space for which $K$ is the unit ball. When $x \in B(K)$ write $\|x\|_{K}$ for its norm.

Let $\mathscr{X}$ be any Banach space; denote its unit ball by $B(\mathscr{X})$. Here we are taking advantage of the coincidence that both "ball" and "Banach" begin with " $B$ ". No confusion should result. Note that $B(B(K))=K$.

The polar of $K$ is defined by

$$
K^{*}=B\left(B(K)^{*}\right)
$$

where $B(K)^{*}$ is the Banach space dual to $B(K)$. Since $B(K)$ is finite dimensional every linear functional is continuous so $(26)$ in fact reduces to the traditional definition

$$
K^{*}=\left\{\xi \in R^{n}:|\langle x, \xi\rangle| \leqq 1 \text { for all } x \in K\right\} .
$$

The support function of $K$ is the norm on $B\left(K^{*}\right)$ (the Minkowski function of $\left.K^{*}\right)$ :

$$
\|\xi\|_{K^{*}}=\psi(\xi) \text {. }
$$

The next theorem formalizes a remark of Herz [24, p. 671].

6.1. ThEOREM. $Z \in \mathscr{Z}^{\dagger}$ if and only if there is an isometry

$$
T: B\left(Z^{*}\right) \rightarrow L^{1}(v)
$$

for some positive measure $v$.

Proof. Suppose $Z=Z_{\mu}=Z_{\mu}^{\dagger}$. For each $\xi \in R^{n}$ let $T \xi$ be the function $\langle, \xi\rangle$ on $S^{n-1}$. When we regard $T$ as a map from $B\left(Z^{*}\right)$ into $L^{1}\left(S^{n-1}, \mu / 2\right)$ it is an isometry because

$$
\begin{aligned}
\|\xi\|_{Z^{*}} & =\psi_{\mu}(\xi)=\frac{1}{2} \int_{S^{n-1}}|\langle x, \xi\rangle| d \mu(x) \\
& =\|T \xi\|_{1} \quad \text { in } L^{1}(\mu / 2) .
\end{aligned}
$$

Conversely, suppose $T: B(K) \rightarrow L^{1}(X, \nu)$ isometrically. We must show $K \in \mathscr{Z} *$, the set of polars of zonoids.

Let $e_{1}, \ldots, e_{n}$ be the usual basis in $\boldsymbol{R}^{n}$ and $f_{i}=T e_{i} \in L^{1}(X, \nu)$. Regard $f=\left(f_{1}, \ldots, f_{n}\right)$ as a map from $X$ into $\boldsymbol{R}^{n}$. For each $\xi \in \boldsymbol{R}^{n}, T \xi(\quad)=\langle f(\quad), \xi\rangle$ maps $X$ into $\boldsymbol{R}$. Finally, define $\mu$ on $\boldsymbol{R}^{n}$ by $\mu=2 \nu \circ f^{-1}$. Then for each $\xi$

$$
\begin{aligned}
\|\xi\|_{K} & =\|T \xi\|_{1}=\int_{X}|\langle f(x), \xi\rangle| d \nu(x) \\
& =\frac{1}{2} \int_{R^{n}}|\langle y, \xi\rangle| d \mu(y)=\|\xi\|_{z_{\dot{\mu}}}
\end{aligned}
$$

Thus $K=Z_{\mu}^{*} \in \mathscr{Z}^{*}$.

The arbitrary measure $\nu$ in Theorem 6.1 can easily be removed since every separable subspace of $L^{1}(X, v)$ is isometric to a subspace of $L^{1}([0,1])=L^{1}$. Finite 
dimensional subspaces are clearly separable. Moreover, we may replace the isometry $T$ by the more geometric sounding:

6.2. CoROllaRy. $K \in \mathscr{Z}^{*}$ if and only if it is a finite dimensional central section of the unit ball of $L^{1}$.

Corollary 2 (p. 493) in [29], which states that every two dimensional Banach space is isometrically embeddable in $L^{1}$, now follows from Corollary 6.2 and Theorem 5.4 .

6.3. Lemma. Let $U: \mathscr{X} \rightarrow \mathscr{Y}$ be a continuous linear map between Banach spaces. Then $U^{*}$ is an isometry if and only if $\mathrm{Cl}(U(B(\mathscr{X})))=B(\mathscr{Y})$.

Proof. Suppose $\eta \in \mathscr{Y}^{*}$. Then

$$
\begin{aligned}
\left\|U^{*} \eta\right\| & =\sup \left\{\left|\left\langle x, U^{*} \eta\right\rangle\right|: x \in B(\mathscr{X})\right\} \\
& =\sup \{|\langle U x, \eta\rangle|: x \in B(\mathscr{X})\}
\end{aligned}
$$

and

$$
\|\eta\|=\sup \{|\langle y, \eta\rangle|: y \in B(\mathscr{Y})\} .
$$

It is clear then that $\left\|U^{*} \eta\right\|=\|\eta\|$ when $\mathrm{Cl}(U(B(\mathscr{X})))=B(\mathscr{Y})$. To prove the converse let $U^{*}$ be an isometry. Since

$$
\|U\|=\left\|U^{*}\right\|=1,
$$

$\mathrm{Cl}(U(B(\mathscr{X}))) \subseteq B(\mathscr{Y})$. Suppose the inclusion is strict and find $y \in B(\mathscr{Y})$ for which $y \notin \mathrm{Cl}(U(B(\mathscr{X})))$. Then the closed convex sets $\{y\}$ and $\mathrm{Cl}(U(B(\mathscr{X})))$ can be separated by some linear functional. That is, there is an $\eta \in \mathscr{Y}^{*}$ for which $\langle y, \eta\rangle$ is greater than the supremum in (29) [14, p. 417, Theorem 10]. Equations (29) and (30) then imply $\left\|U^{*} \eta\right\|<\|\eta\|$, a contradiction.

6.4. THEOREM. $Z$ is a zonoid if and only if it is the closure of a (norm) continuous linear image of the ball $B\left(L^{\infty}\right)$ or, equivalently, a weak-* continuous linear image of that ball.

Proof. Suppose $Z$ is a zonoid. Then there is an isometry $T: B(Z)^{*} \rightarrow L^{1}$. Since $B(Z)^{*}$ is finite dimensional it is reflexive so $T^{* *}=T$ is also an isometry. Then apply Lemma 6.3 to $U=T^{*}$ to deduce

$$
\mathrm{Cl}\left(T^{*}\left(B\left(L^{\infty}\right)\right)\right)=Z .
$$

Since $T^{*}$ is weak-* continuous and $B\left(L^{\infty}\right)$ is weak-* compact its image under $T^{*}$ is compact and hence closed; the closure in (31) is unnecessary.

Conversely, suppose $U: L^{\infty} \rightarrow \boldsymbol{R}^{n}$ is linear, surjective and norm continuous with respect to any (hence every) vector space topology on $\boldsymbol{R}^{n}$. Let $K=\mathrm{Cl}\left(U\left(B\left(L^{\infty}\right)\right)\right)$. Lemma 6.3 implies that $U^{*}: B(K)^{*} \rightarrow\left(L^{\infty}\right)^{*}$ is an isometry. Since $L^{\infty}=\left(L^{1}\right)^{*}$ is an abstract $M$-space its dual is isometric to $L^{1}(\nu)$ for some measure $\nu[26$, p. 1021, 
Theorem 15] and [25, p. 532, Theorem 7]. Theorem 6.1 then implies $K$ is a zonoid. If $U$ happens to be weak-* continuous then, as we noted above, $U\left(B\left(L^{\infty}\right)\right)=K$.

Theorem 6.4 may be thought of as a continuous analogue of part 4 of Theorem 3.3. It is the heart of Lindenstrauss's elegant proof (in [30]) of Liapounoff's convexity theorem. There is also a discrete version of Corollary 6.2.

Let

$$
l_{n}^{p}=\left\{\left.\left(x_{1}, \ldots, x_{n}\right)|| x_{1}\right|^{p}+\cdots+\left|x_{n}\right|^{p} \leqq 1\right\}
$$

for $1 \leqq p<\infty$ and $l_{n}^{\infty}=\left\{\left(x_{1}, \ldots, x_{n}\right)|\max | x_{i} \mid \leqq 1\right\}$.

6.5. TheOREM. A polytope $K$ is the polar of a zonoid if and only if it is a central section of a generalized cross polytope.

Proof. If $K^{*}$ is a zonotope it is a linear image of a cube (Theorem 3.3, part 4). If we center $K^{*}$ at 0 we can assume that $K^{*}=T\left(l_{N}^{\infty}\right)$. Since $T: R^{N} \rightarrow R^{n}$ is surjective its adjoint $T^{*}: \boldsymbol{R}^{n} \rightarrow \boldsymbol{R}^{N}$ is injective and maps $K$ bijectively onto a central section of $l_{N}^{1}$ (Lemma 6.3).

Conversely, a generalized cross polytope is the unit ball of a subspace of $L^{1}$, so each of its central sections enjoys that property. The theorem then follows from Corollary 6.2.

Theorem 5.4 shows that it is rare for a polytope to be an image of $l_{N}^{\infty}$ or a section of $l_{M}^{1}$. Compare this with a result of Neumann [34]: every centrally symmetric polytope is an image of some $l_{N}^{1}$ and a section of some $l_{M}^{\infty}$.

6.6. THEOREM. $l_{n}^{p}$ is a zonoid when $n \geqq 3$ and $2 \leqq p \leqq \infty$.

Proof. In [24] Herz observes that the Minkowski function of $l_{n}^{q}$ is negative definite and homogeneous when $1 \leqq q \leqq 2$. The theorem then follows from Theorem 2.7, Corollary 2.6, equation (28) and the fact that $\left(l_{n}^{q}\right)^{*}=l_{n}^{p}$ when $p^{-1}+q^{-1}=1$.

Note that $l_{n}^{\infty}$ is a hypercube and hence a zonotope while $l_{n}^{2}$ is a ball. Thus we have long known the extreme cases of Theorem 6.6. We have already noted that the cross polytope $l_{n}^{1}$ is not a zonoid for $n \geqq 3$. Since the set of nonzonoids is open in $\mathscr{K}$ (Theorem 5.3) and $p \leadsto l_{n}^{p}$ is a continuous map from $[1, \infty]$ to $\mathscr{K}$ we know $l_{n}^{p}$ is not a zonoid for $p$ sufficiently close to 1 . (See $[29$, p. 494] for another proof of this remark.)

6.7. Conjecture. $l_{n}^{p}$ is not a zonoid for $n \geqq 3$ and $1 \leqq p<2$.

In [35] Paley proved that the Banach space $l^{q}$ is not isomorphic to a subspace of $L^{1}$ for $2 \leqq q \leqq \infty$. Here "isomorphism" means "continuous, bijective, linear map." Isomorphisms need not be isometries. Conjecture 6.7 is a finite dimensional version of Paley's result with “isometric" replacing "isomorphic". Using Paley's techniques Rosenthal has shown that $l_{n}^{p}$ is not a zonoid for

$$
\begin{aligned}
& p \leqq 2 \log 3 / \log 7, \quad n=3, \\
& p \leqq 2 \log n /(\log n+\log 3), \quad n>3 .
\end{aligned}
$$


As $n \rightarrow \infty$ the right member of (32) approaches 2; this is further evidence for Conjecture 6.7.

Conjecture 6.7 would follow from Lemma 5 of [29] (p. 494) if we could show that the projection constant of $l_{n}^{p}$ was a monotonic function of $p$. (See Grünbaum, [20], for facts about projection constants.)

6.8. Conjecture. If both $Z$ and $Z^{*}$ are $n$ dimensional zonoids ( $n \geqq 3$ ) then $Z$ is an ellipsoid.

The truth of 6.8 would imply that of 6.7. Grothendieck proved that if an infinite dimensional Banach space is isomorphic to a subspace of $L^{1}$ and a quotient space of $L^{\infty}$ then it is isomorphic to Hilbert space. (For a proof see [31, Theorem 6.2].) That result is to Conjecture 6.8 as Paley's is to Conjecture 6.7: Conjecture 6.8 is a finite dimensional analogue with "isomorphic" replaced by "isometric."

Next we shall prove Conjecture 6.8 for polytopes. Along the way we will almost characterize the polars of the zonotopes. Let $K$ be an $n$ dimensional polytope symmetric about 0 . For each face $F$ of $K$ let

$$
F^{\wedge}=\{\xi \mid\langle x, \xi\rangle=1 \text { when } x \in F\} .
$$

Then $F \leadsto F^{\wedge}$ is an inclusion reversing bijection between the set of faces of $K$ and the corresponding set for $K^{*}\left(\left[21\right.\right.$, p. 47]). $F^{\wedge \wedge}=F$.

$$
\operatorname{dim} F^{\wedge}=n-1-\operatorname{dim} F .
$$

An $r$ dimensional face $F$ of $K$ lies in a unique $r+1$ dimensional subspace $V(F)$ of $\boldsymbol{R}^{n}$ and in a translate of a unique $r$ dimensional subspace $W(F)$ of $\boldsymbol{R}^{n}$. We shall say $K$ is $r$-equatorial if for every such $F, V(F) \cap \partial K$ is a union of $r$ dimensional faces. Every $K$ under consideration is trivially $(n-1)$-equatorial. Central symmetry implies $K$ is 0 -equatorial. The octahedron is 1-equatorial since every edge lies on an "equator" of edges. The cube does not enjoy this property.

\subsection{LEMMA.}

$$
V\left(F^{\wedge}\right)=W(F)^{\perp} .
$$

Proof. Let $x$ be an interior point of $F$. Then for all $w \in W(F)$ with $\|w\|$ sufficiently small, $x+w \in F$. Suppose $\xi \in \hat{F}$. Then

$$
\langle x, \xi\rangle=\langle x+w, \xi\rangle=1
$$

implies $\langle w, \xi\rangle=0$. Thus $\xi \in W(F)^{\perp}$. The lemma then follows from a dimension count. Note that ${ }^{\wedge}$ and ${ }^{\perp}$ may be moved at will to either side of (35).

6.10. LeMmA. $K$ is $r$-equatorial if and only if the $n-r-1$ dimensional faces of the $n-r$ dimensional faces of $K^{*}$ occur in parallel pairs.

Proof. Note that the lemma is true when $r=0$ or $n-1$ because $K$ and hence $K^{*}$ is centrally symmetric, so suppose $1 \leqq r \leqq n-2$. 
Let $F^{\wedge}$ be an $n-r$ dimensional face of $K^{*}$. Let $G^{\wedge}$ be one of its $n-r-1$ dimensional faces; then $F$ is an $r-1$ dimensional face of the $r$ dimensional face $G$ of $K$. If $K$ is $r$-equatorial, $V(G) \cap \partial K$ is a union of $r$ dimensional faces. Then just two of them, $G$ and $G^{\prime}$, have $F$ for a face. Since $W(G)=V(G)^{\perp}=V\left(G^{\prime}\right)^{\perp}=W\left(G^{\wedge}\right)^{\perp}, G$ and $G^{\wedge}$ are parallel faces of $F^{\wedge}$.

Conversely, if given $F^{\wedge}$ and $G^{\wedge}$ we can always find a face $G^{\prime \curlywedge}$ of $F^{\wedge}$ parallel to $G^{\wedge}$ the above argument shows $F$ is in the interior of $V(G) \cap \partial K$. It follows that the union $M$ of the $r$ dimensional faces of $K$ in $V(G) \cap \partial K$ is open and closed. Since $V(G)$ is $r+1$ dimensional and $r \geqq 1, V(G) \cap \partial K$ is connected and hence exhausted by $M$. Thus $K$ is $r$-equatorial.

It follows from Lemma 3.4 that if $K$ is a convex body with summands [0,v] and $[0, w]$ and $v$ is not a multiple of $w$ then $K$ has $[0, v]+[0, w]$ as a summand. Hence any $K$ has a maximum zonotopic summand $Z(K)$. Here "maximum" may be thought of relative to the ordering by inclusion or by "divisibility" in $\mathscr{K}^{+} ; Z(K)$ is the same in either case. $K$ is a zonotope when $Z(K)=K$.

6.11. THEOREM. The following conditions on an $n$ dimensional convex polytope $K$ centered at 0 in $\boldsymbol{R}^{n}$ are equivalent.

1. $K$ is r-equatorial, $0 \leqq r \leqq n-1$.

2. $K$ is $(n-2)$-equatorial.

3. The edges of the two dimensional faces of $K^{*}$ occur in parallel pairs.

4. $Z\left(K^{*}\right)$ has a zone parallel to every edge of $K^{*}$.

Proof. $(1 \Rightarrow 2)$ is obvious.

$(2 \Rightarrow 3)$ is a special case of Lemma 6.10 .

$(3 \Rightarrow 4)$. Let $v$ be an edge of $K^{*}$. Then an argument similar to that in $(5 \Rightarrow 3)$ of Theorem 3.3 shows that $\lambda_{0}=\lambda_{0}\left(K^{*}, v\right)>0$, so that $Z\left(K^{*}\right)$ has a zone parallel to $v$.

$(4 \Rightarrow 1)$. If $Z\left(K^{*}\right)$ satisfies 4 then (14) implies that the $r$ dimensional faces of $K^{*}$ share with those of $Z\left(K^{*}\right)$ the property of having $r-1$ dimensional faces in parallel pairs. Lemma 6.10 then shows $K$ satisfies 1 .

\subsection{COROLlaRY. If $Z$ is a zonotope then $Z^{*}$ is $r$-equatorial, $0 \leqq r \leqq n-1$.}

Unfortunately, the converse of Corollary 6.12 is false. A truncated octahedron $K$ satisfies 3 and hence $K^{*}$ satisfies 1 in Theorem 6.11 but $K$ will not be a zonotope unless the truncation points divide the edges into three equal segments (see $\S 3$ ). In general $Z(K)$ has 6 zones and $K=Z(K)+K^{\prime}$ where $K^{\prime}$ is an octahedron.

6.13. THEOREM. If $Z$ is an $n$ dimensional zonotope $(n \geqq 3)$ then $Z^{*}$ is not.

Proof. It suffices to find a two dimensional face of $Z^{*}$ which is not centrally symmetric. Let $F$ be a three dimensional face of $Z^{*}$; since $Z^{*}$ is 3- and 1-equatorial, $F$ is 1-equatorial. We shall show that a 1-equatorial 3 dimensional polyhedron $F$ cannot have only centrally symmetric faces. Let $p$ be a vertex of $F$. The sum of the 
angles at $p$ is less than $2 \pi$. Since $F$ is 1-equatorial it follows that the sum of two adjacent angles at $p$ is less than $\pi$. Now let $E$ be the edge of $F$ joining vertices $p_{1}$ and $p_{2}$. $E$ lies on two faces $A$ and $B$. Let $\alpha_{1}$ be the angle at the vertex $p_{1}$ of $A$; similarly define $\alpha_{2}, \beta_{1}$ and $\beta_{2}$. Then

$$
\alpha_{i}+\beta_{i}<\pi, \quad i=1,2,
$$

since $\alpha_{i}$ and $\beta_{i}$ are adjacent angles at $p_{i}$. Thus $\alpha_{1}+\alpha_{2}+\beta_{1}+\beta_{2}<2 \pi$ so either $\alpha_{1}+\alpha_{2}<\pi$ or $\beta_{1}+\beta_{2}<\pi$. The first (second) inequality implies $A(B)$ is not centrally symmetric.

Since a polytope is never an ellipsoid Theorem 6.13 is in fact Conjecture 6.8 for polytopes. Theorem 6.13 can also be proved combinatorially by counting faces and vertices of zonotopes; this idea is Perles's.

7. Brightness. Let $K \in \mathscr{K}$ have a nonempty interior; only such convex bodies will concern us in this section. For each Borel subset $E$ of $S^{n-1}$ define $\mu=\mu_{K}$ by

$$
\mu(E)=\text { area of } \bigcup\left\{F_{\xi} \mid \xi \in E\right\} .
$$

$\mu$ is called the surface area measure of $K . \mu$ is a measure and it is easy to see that $\tilde{\mu}\left(S^{n-1}\right)=0$ and that $\mu\left(H_{\xi}^{\circ}\right)>0$ for all $\xi$.

Conversely, any such measure is the surface area measure of a $K \in \mathscr{K}$ which is unique to within translation. We shall follow Firey [17] and call this result Minkowski's Theorem; Minkowski knew it for polytopes. A proof of the general theorem can be found in Fenchel and Jessen, [16, p. 15].

The brightness $\beta(\xi)$ of $K$ in the direction $\xi \in S^{n-1}$ is the area of the orthogonal projection of $K$ on $\xi^{\perp}$ :

$$
\beta(\xi)=\frac{1}{2} \int|\langle x, \xi\rangle| d \mu_{K}(x)=\psi_{\mu_{K}}(\xi)
$$

$[17$, p. 9 , equation (6)].

The convex set $Z_{\mu_{K}}^{*}$ is the projection body of $K$; its radius in the direction $\xi$ is $\beta(\xi)=\psi_{\mu_{K}}(\xi)$. The remarks above show

\subsection{THEOREM. $K \in \mathscr{Z} *$ if and only if $K$ is a projection body.}

Herz remarked that the first part of Theorem 5.4 follows easily from Theorem 7.1: every two dimensional element of $\mathscr{K}^{\dagger}$ is a projection body [24, p. 672].

The brightness function can be redefined and generalized using mixed volumes; analogues of the next theorem are true. Details appear in [9], [17] and [40].

Let $\mu$ be a measure on $S^{n-1}$; suppose no proper subspace of $\boldsymbol{R}^{n}$ supports $\mu$. We wish to consider the "symmetric part" $\mu^{s}$ of $\mu$ relative to the antipodal map:

$$
\mu^{s}(E)=\frac{1}{2}(\mu(E)+\mu(-E)) \text {. }
$$

Then

$$
\begin{gathered}
\mu^{s} \circ \pi^{-1}=\mu \circ \pi^{-1}, \\
\mu^{s}(E)=\frac{1}{2} \mu^{s} \circ \pi^{-1}(\pi(E)) \quad \text { when } E \cap(-E)=\varnothing
\end{gathered}
$$


and $\mu=\mu^{s}$ if and only if $\mu$ is the surface area measure of a centrally symmetric convex body. Moreover,

$$
Z_{\mu}^{\dagger}=Z_{\mu^{s}}
$$

7.2. TheOREM. A centrally symmetric convex body is determined up to a translation by its brightness.

Proof. Suppose $K$ and $L$ are centrally symmetric convex bodies with $\beta_{K}=\beta_{L}$. Then (37) implies $Z_{\mu_{K}}=Z_{\mu_{L}}$ and hence $\mu_{K} \circ \pi^{-1}=\mu_{L} \circ \pi^{-1}$ (Corollary 2.9). Equation (40) and the central symmetry of $K$ and $L$ show $\mu_{K}=\mu_{L}$. Then $K$ is a translate of $L$ by virtue of Minkowski's Theorem.

7.3. COROllary. The only centrally symmetric body of constant brightness is a ball.

Theorem 7.2 is due to Alexandrov [2]; another proof can be found in [17, p. 11] and a generalization in [9]. We have just deduced it from Theorem 2.8 by way of Corollary 2.9. The argument works in reverse as well:

\subsection{THEOREM. Theorem 7.2 implies Theorem 2.8 .}

Proof. Suppose $Z_{\mu}$ is a translate of $Z_{v}$.

Let $K$ and $L$ be convex bodies with surface area measures $\mu^{s}$ and $\nu^{s}$ respectively; $K$ and $L$ are centrally symmetric so each is uniquely determined when we demand that its center be 0 . Now Corollary 2.4 and (37) imply $K$ and $L$ have the same brightness so Alexandrov's Theorem (7.2) tells us $K=L$. Thus $\mu^{s}=\nu^{s}$ so (39) implies $\mu \circ \pi^{-1}=\nu \circ \pi^{-1}$.

We have proved the harder of the two implications of Corollary 2.9. The other proceeds as before. We already noted that Corollary 2.9 and Theorem 2.8 are equivalent, so Theorem 7.4 has been proved.

Zonoids tend to be dim. Call a $K \in \mathscr{K}$ brighter than $L$ if for all $\xi, \beta_{K}(\xi)>\beta_{L}(\xi)$. Then Schneider and Petty proved, independently, that if a zonoid $Z$ is brighter than $K \in \mathscr{K}$ it is because the volume of $Z$ exceeds that of $K([40$, p. 75, Satz 2], and [36]). Thus for zonoids an overall increase in brightness requires an increase in volume. That fails for nonzonoids. If $K \notin \mathscr{Z}$ then there is an $L \in \mathscr{K}$ which is smaller in volume but brighter [40, p. 80, Satz $3 \mathrm{ff}$.]. $L$ cannot be a zonoid, of course. These two results together do not quite characterize zonoids.

\section{REFERENCES}

1. A. D. Alexandrov, A theorem on convex polyhedra, Trudy Mat. Inst. Steklov. Sect. Math. 4 (1933), 87. (Russian)

2. -__, Zur Theorie der gemischten Volumina von Konvexen Körpern, II, Neue Ungleichungen zwischen den gemischten Volumina und ihre Anwendungen, Mat. Sb. 2 (1937), 1205-1238. (Russian)

3. W. Blaschke, Vorlesungen über Differential-geometrie. Vol. II, Affine Differentialgeometrie, Springer-Verlag, Berlin, 1923. 
4. W. Blaschke, Kreis und Kugel, 2nd ed., de Gruyter, Berlin, 1956.

5. W. Blaschke and K. Reidemeister, Über die Entwicklung der Affingeometrie, Jber Deutsch. Math.-Verein. 31 (1922), 63-82.

6. E. Bolker, Functions resembling quotients of measures, Trans. Amer. Math. Soc. 124 (1966), 292-312.

7. T. Bonnesen and W. Fenchel, Theorie der konvexen Körper, Ergebnisse der Mathematik, Vol. 3, Springer, Berlin, 1934.

8. J. J. Burckhardt, Über konvexe Körper mit Mittelpunkt, Viertlejschr. Natur. Ges. Zürich 85 (1940), 149-154.

9. G. D. Chakerian, Sets of constant relative width and constant relative brightness, Trans. Amer. Math. Soc. 129 (1967), 26-37.

10. G. Choquet, Mesures coniques, affines et cylindriques: Structure et opérations, C. R. Acad. Sci. Paris 266 (1968), 567-569.

11. —_- Mesures coniques et affines invariants par isométries. Zonoformes, zonoèdres et fonctions de type negatif, C. R. Acad. Sci. Paris 266 (1968), 619-621.

12. H. S. M. Coxeter, The classification of zonohedra by means of projective diagrams, J. Math. Pures Appl. (9) 41 (1962), 137-156.

13. - - Regular polytopes, 2nd ed., Macmillan, New York, 1963.

14. N. Dunford and J. Schwartz, Linear operators. Part I. General theory, Pure and Appl. Math., vol. 7, Interscience, New York, 1958.

15. H. G. Eggleston, Convexity, Cambridge Tracts in Math. and Math. Phys., no. 47, Cambridge Univ. Press, New York, 1958.

16. W. Fenchel and B. Jessen, Mengenfunctionen und konvexe Körper, Det. Kgl. Danske Vid. Selsk. Math.-Fys. Medd. 3 (1938).

17. W. J. Firey, The brightness of convex bodies, Technical Report No. 19, Department of Mathematics, Oregon State University, Corvallis, Oregon, 1965.

18. _- , Blaschke sums of convex bodies and mixed bodies, Proc. Colloq. on Convexity, (Copenhagen, 1965), Kobenhavns Univ. Mat. Inst., Copenhagen, 1967, pp. 94-101.

19. S. Gelbart, $L^{1}$-unit balls and conical measures, Proc. Amer. Math. Soc. (to appear).

20. B. Grünbaum, Projection constants, Trans. Amer. Math. Soc. 95 (1960), 451-465.

21. - C Convex polytopes, Wiley, New York, 1967.

22. P. R. Halmos, The range of a vector measure, Bull. Amer. Math. Soc. 54 (1948), 416-421.

23. - Measure theory, Van Nostrand, Princeton, N. J., 1950.

24. C. S. Herz, A class of negative definite functions, Proc. Amer. Math. Soc. 14 (1963), 670-676.

25. S. Kakutani, Concrete representation of abstract $(L)$-spaces and the mean ergodic theorem, Ann. of Math. (2) 42 (1941), 523-537.

26. - Concrete representation of abstract $(M)$-spaces (A characterization of the space of continuous functions), Ann. of Math. (2) 42 (1941), 994-1024.

27. R. P. Kaufman and N. W. Rickert, An inequality concerning measures, Bull. Amer. Math. Soc. 72 (1966), 672-676.

28. A. Liapounoff, Sur les fonctions-vecteurs complètement additives, Bull. Acad. Sci. URSS Ser. Math. 4 (1940), 465-478. (Russian)

29. J. Lindenstrauss, On the extension of operators with a finite-dimensional range, Illinois $\mathbf{J}$. Math. 8 (1964), 488-499.

30. —_ A A short proof of Liapounoff's convexity theorem, J. Math. Mech. 15 (1966), 971-972.

31. J. Lindenstrauss and A. Pełczyński, Absolutely summing operators in $\mathscr{L}_{p}$ spaces and their applications, Studia Math. 29 (1968), 275-326. 
32. N. Lindquist, Representations of central convex bodies, Thesis, Oregon State University, Corvallis, Oregon, 1968.

33. A. E. Mayer, Grösste Polygone mit gegebenen Seitenvektoren, Comment. Math. Helv. 10 (1938), 288-301.

34. H. Neumann, Beliebige Konvexe Polytope als Schnitte und Projectionen hoherdimensionaler Würfel, Simplizes und Masspolytope, Math. Z. 65 (1965), 91-103.

35. R. E. A. C. Paley, Some theorems on abstract spaces, Bull. Amer. Math. Soc. 42 (1936), 235-240.

36. C. M. Petty, Projection bodies, Proc. Colloq. on Convexity, (Copenhagen, 1965), Kobenhavns Univ. Mat. Inst., Copenhagen, 1967, pp. 234-241.

37. N. W. Rickert, The range of a measure, Bull. Amer. Math. Soc. 73 (1967), 560-563.

38. __- Measures whose range is a ball, Pacific J. Math. 23 (1967), 361-371.

39. D. Rutovitz, Some parameters associated with finite-dimensional Banach spaces, J. London Math. Soc. 40 (1965), 241-255.

40. R. Schneider, Zu einem Problem von Shephard über die Projectionen konvexer Körper, Math. Z. 101 (1967), 71-82.

41. —_, Über eine Integralgleichung in der Theorie der konvexen Körper, Math. Nachr. (to appear).

42. G. Schwarz, Variations on vector measures, Pacific J. Math. 23 (1967), 373-375.

43. Z. Semadeni, Generalizations of Bohr's theorem on Fourier series with independent characters, Studia Math. 23 (1963), 159-179.

44. G. C. Shephard, Decomposable convex polyhedra, Mathematika 10 (1963), 89-95.

45. —_, Approximation problems for convex polyhedra, Mathematika 11 (1964), 9-18.

46. _-_ Polytopes with centrally symmetric faces, Canad. J. Math. 19 (1967), 1206-1213.

47. G. Choquet, Mesures coniques, affines et cylindriques, Instituto Nazionale di Alta Matematica, Symposia Matematica, Vol. II, Gubbio, 1968.

\author{
UNIVERSITY OF CALIFORNIA, \\ Berkeley, California \\ Bryn MaWr College, \\ Bryn Mawr, Pennsylvania
}

